\title{
A Single Intra-PFC Infusion of BDNF Prevents Cocaine- Induced Alterations in Extracellular Glutamate within the Nucleus Accumbens
}

\author{
William J. Berglind, Timothy W. Whitfield Jr, Ryan T. LaLumiere, Peter W. Kalivas, and Jacqueline F. McGinty \\ Department of Neurosciences and Neurobiology of Addiction Research Center, Medical University of South Carolina, Charleston, South Carolina 29425
}

The glutamatergic pathway arising in the dorsomedial prefrontal cortex $(\mathrm{dmPFC})$ and projecting to the nucleus accumbens $(\mathrm{NAc})$ core is a critical component of the reward circuitry that underlies reinstatement to cocaine-seeking behavior. Brain-derived neurotrophic factor (BDNF) is expressed by and modulates PFC-NAc neurons. BDNF infusion into the dmPFC attenuates reinstatement to cocaine-seeking behavior, as well as some cocaine-induced molecular adaptations within the NAc. In the present study, it is demonstrated that a single intra-dmPFC infusion of BDNF prevents cocaine self-administration-induced reduction in basal extracellular glutamate, as well as cocaine prime-induced increases in extracellular glutamate levels within the NAc. These data suggest that intra-PFC BDNF attenuates reinstatement to cocaine-seeking behavior by normalizing cocaine-induced neuroadaptations that alter glutamate neurotransmission within the NAc.

\section{Introduction}

Relapse to drug seeking during abstinence from cocaine is marked by disturbances in glutamatergic neurotransmission in the prefrontal cortex (PFC)-nucleus accumbens (NAc) pathway in animal models of addiction (Kalivas and Volkow, 2005). Reinstatement of cocaine seeking is blocked by the inactivation of the dorsomedial PFC (dmPFC) or NAc core by GABA agonists or the $\mathrm{Na}^{+}$channel blocker, tetrodotoxin (TTX) (McFarland and Kalivas, 2001; Capriles et al., 2003; McLaughlin and See, 2003; Fuchs et al., 2004, 2005). Two different mechanisms control basal and cocaine-evoked extracellular glutamate levels: basal levels are TTX-independent and cystine/glutamate antiporter (xc-)dependent, whereas cocaine-evoked levels are action potential and TTX-dependent (Pierce et al., 1996; Baker et al., 2003). Basal glutamate levels arise from nonvesicular glutamate release that stimulates extrasynaptic pools of group II metabotropic glutamate receptors (mGluRs) (Baker et al., 2002; Xi et al., 2002; Moran et al., 2005). Extrasynaptic group II mGluRs inhibit vesicular glutamate release (Losonczy et al., 2003). After repeated cocaine exposure, diminished $\mathrm{xc}$-function results in a decrease in basal extracellular glutamate and diminished mGluR-mediated inhibition of synaptic glutamate release (Baker et al., 2003; Moran et al., 2005).

The molecular substrates that underlie cocaine-induced alterations in PFC-NAc synapses remain to be elucidated; however, there is evidence that brain-derived neurotrophic factor (BDNF)

Received Nov. 12, 2008; revised Jan. 23, 2009; accepted Feb. 10, 2009.

This work was supported by National Institutes of Health Grants P50 DA015369, F31 DA018500, F31 DA023743, F32 DA021460, and T32 DA07288.

Correspondence should be addressed to Jacqueline F. McGinty, Department of Neurosciences, Medical University of South Carolina, 173 Ashley Avenue, MSC 510, Charleston, SC 29425. E-mail: mcginty@musc.edu.

DOI:10.1523/JNEUROSCI.5457-08.2009

Copyright $\odot 2009$ Society for Neuroscience $\quad$ 0270-6474/09/293715-05\$15.00/0 is an important contributor. BDNF activity is associated with synaptic plasticity and related changes in receptor-mediated intracellular signaling that depend on the interaction of BDNF with tyrosine-related kinase B (TrkB) receptors ( $\mathrm{Lu}, 2003)$. For example, BDNF is able to exert long-term adaptations by facilitating long-term potentiation and enhancing postsynaptic responses. Furthermore, in vitro studies of mature cortical cultures have shown that BDNF increases the expression and translation of mRNAs that are associated with synaptic plasticity (Schratt et al., 2004). Finally, exogenous application of BDNF enhances glutamatergic transmission in the cerebral cortex by facilitating glutamate presynaptic release as well as by enhancing AMPA receptor responsiveness (Lessmann, 1998).

We found that conditioned cue-induced or cocaine-induced reinstatement after extinction from cocaine self-administration is significantly attenuated in rats that receive an infusion of BDNF into the dmPFC shortly after the last cocaine self-administration session (Berglind et al., 2007). Furthermore, an intra-PFC BDNF infusion prevented a decrease in phospho-extracellular signalregulated protein kinase (ERK) expression in the NAc that occurs after $22 \mathrm{~h}$ of abstinence from cocaine self-administration (Berglind et al., 2007). This evidence suggests that intra-PFC BDNF suppresses reinstatement behavior by altering cocaine-induced neuroadaptations in the PFC-NAc pathway. The present study examined the effects of intra-PFC BDNF infusions on basal and cocaine-induced extracellular glutamate levels in the NAc in rats with a cocaine self-administration history.

\section{Materials and Methods}

Animals. Male Sprague Dawley rats $(N=56$; experiment $1, N=28$; experiment 2, $N=28$; Charles River Laboratories), weighing 275-325 g at the time of surgery, were housed individually on a reverse light/dark cycle. Rats were maintained on 20-25 g of rat chow per day, with water available ad libitum. All protocols were approved by the Institutional 
Animal Care and Use Committee of the Medical University of South Carolina and performed in accordance with the National Institutes of Health (NIH) Guide for the Care and Use of Laboratory Animals (NIH publications number 80-23, revised 1996).

Lever response training. All rats were trained to lever press on a fixed ratio 1 schedule of food reinforcement ( $45 \mathrm{mg}$ pellets; Noyes) in soundattenuated operant conditioning chambers (MED Associates) during a $16 \mathrm{~h}$ overnight training session as described previously (Berglind et al., 2007). After food training, food pellet dispensers were removed from the chambers.

Surgery. After food training ( $48 \mathrm{~h})$, rats were anesthetized with ketamine hydrochloride (66 mg/kg, i.p.; Fort Dodge Animal Health) and xylazine ( $1.33 \mathrm{mg} / \mathrm{kg}$, i.p.; Bayer) followed by equithesin $(0.5 \mathrm{ml} / \mathrm{kg}$, i.p.) solution. Catheters constructed of SILASTIC tubing $(0.64 \mathrm{~mm}$ inner diameter, $1.19 \mathrm{~mm}$ outer diameter; Dow Corning) were implanted into the right jugular vein of rats. Then rats were mounted onto a stereotaxic device (Stoelting), and bilateral stainless steel cannulae (26 gauge; Plastics One) were implanted $1 \mathrm{~mm}$ above the $\mathrm{dmPFC}$ target region [anterior-posterior (AP), +3.0; medial-lateral (ML), +0.6; dorsoventral, -1.6 , relative to bregma) (Paxinos and Watson, 1998), after which bilateral stainless steel cannulae (20 gauge; Plastics One) were implanted $2 \mathrm{~mm}$ above the NAc target region (AP, 2.5 ; ML, $0.66^{\circ}$, relative to bregma) (Paxinos and Watson, 1998). Cranioplastic cement anchored by three steel machine screws was applied to permanently secure the dmPFC and NAc cannulae to the skull.

Cocaine self-administration. Self-administration was conducted during $2 \mathrm{~h}$ daily sessions during the rats' dark cycle and continued until the rats self-administered a minimum of 10 cocaine (cocaine hydrochloride; $0.2 \mathrm{mg} / 0.05 \mathrm{ml}$; National Institute on Drug Abuse) infusions per session on an FR1 schedule of reinforcement for a minimum of $10 \mathrm{~d}$ (acquisition criterion). After each infusion, responses on the active lever had no consequences during a $20 \mathrm{~s}$ timeout period. Responses on the inactive lever had no programmed consequences. Yoked-saline rats in experiment 2 received an infusion of saline $(0.05 \mathrm{ml}$ bolus $)$ over $2 \mathrm{~s}$, contingent on the cocaine infusion received by the self-administering rat in the adjacent chamber. Rats underwent $10 \mathrm{~d}$ of cocaine self-administration (experiments 1 and 2) or yoked-saline (experiment 2) infusions before intracranial infusion.

dmPFC intracranial infusions. An infusion protocol and dose of BDNF $(0.75 \mu \mathrm{g} / \mathrm{side})$ previously demonstrated to alter cocaine seeking (Lu et al., 2004; Berglind et al., 2007) were used. Immediately after the last selfadministration session in experiments 1 and 2, infusion cannulae (33 gauge; Plastics One) were inserted bilaterally into the guide cannulae, such that $1 \mathrm{~mm}$ of the infusion cannulae extended past the end of the guide cannulae. Human recombinant BDNF (R\&D Systems) or vehicle (10 mm sterile PBS) were infused bilaterally into the dmPFC using gastight Hamilton syringes $(10 \mu \mathrm{l})$ and an infusion pump (Harvard Apparatus). A volume of $0.5 \mu \mathrm{l}$ was infused over $2 \mathrm{~min}$, and the infusion cannulae remained in the guide cannulae for $1 \mathrm{~min}$ before and after the infusion.

Extinction and reinstatement. For experiment 1, beginning $1 \mathrm{~d}$ after self-administration, the rats underwent $10 \mathrm{~d}$ of extinction training. During each $2 \mathrm{~h}$ session, lever presses had no programmed consequences. After extinction, the rats underwent a single $2 \mathrm{~h}$ cocaine prime $(10 \mathrm{mg} / \mathrm{kg}$, i.p.)-induced reinstatement test during which lever presses were recorded but had no programmed consequences. After the reinstatement test, the rats underwent $7-10 \mathrm{~d}$ of additional extinction training before undergoing microdialysis. For experiment 2, rats underwent $14 \mathrm{~d}$ of extinction training before their first dialysis day and a further $7 \mathrm{~d}$ of extinction training before their second day of dialysis performed in the contralateral hemisphere. No-net flux in vivo microdialysis was performed on both dialysis days in experiment 2 (see below).

Experiment 1: in vivo microdialysis. Rats with a cocaine selfadministration history infused with vehicle in the dmPFC (COC-VEH) or rats with a cocaine self-administration history infused with BDNF in the dmPFC (COC-BDNF) were included in this experiment. Microdialysis probes were constructed using fused silica for inlet and outlet tubing inserted into 26-gauge cannulae topped with a semipermeable membrane with $2 \mathrm{~mm}$ of active length as described previously (Baker et al.,
2003). On the evening before the sample collection, the rats were housed in the operant chambers, and probes were inserted into the NAc via the previously implanted 20-gauge cannulae. Dialysis buffer ( $5 \mathrm{~mm}$ glucose, $2.5 \mathrm{~mm} \mathrm{KCl}, 140 \mathrm{~mm} \mathrm{NaCl}, 1.4 \mathrm{~mm} \mathrm{CaCl}_{2}, 1.2 \mathrm{~mm} \mathrm{MgCl}_{2}$, and 0.15\% PBS, $\mathrm{pH} 7.4$ ) was perfused through the probe overnight at $0.2 \mu \mathrm{l} /$ minute. In the morning, the flow rate was increased to $2.0 \mu \mathrm{l} /$ minute for $2 \mathrm{~h}$. Each rat then received an intraperitoneal injection of cocaine $(10 \mathrm{mg} / \mathrm{kg})$; then samples were collected every $10 \mathrm{~min}$ for an additional $2 \mathrm{~h}$. All samples were collected into $10 \mu \mathrm{l}$ of $0.05 \mathrm{M} \mathrm{HCl}$ and stored at $-80^{\circ} \mathrm{C}$ for subsequent analysis of extracellular glutamate (see below).

Experiment 2: no-net flux microdialysis. There were four groups in this experiment: rats that had a cocaine history that were infused with vehicle (COC-VEH) or BDNF (COC-BDNF) and rats with a yoked-saline history that were infused with vehicle (SAL-VEH) or BDNF (SAL-BDNF). No-net flux microdialysis was performed in the same manner as described above, except that the rats were not returned to the operant chambers; they were placed into microdialysis chambers in a separate room. After collection of baseline samples for $2 \mathrm{~h}$, the no-net flux procedure began by changing the perfusate to dialysis buffer containing $2.5,5$, or $10 \mu \mathrm{M}$ glutamate. Liquid switches were used to minimize the pressure fluctuations while changing dialysis buffers containing varying concentrations of glutamate. Six samples were collected at $10 \mathrm{~min}$ intervals for each concentration.

Analysis and quantification of extracellular glutamate. Glutamate levels from the NAc were analyzed using HPLC as described previously (Bell et al., 2000). Samples were loaded on an autosampler (Gilson Medical Electric) that derivatized the sample with $o$-pthalaldehyde before injecting it into the column via circulating mobile phase ( $100 \mathrm{~mm}$ sodium dihydrogen phosphate monobasic, $90 \mathrm{M} \mathrm{EDTA}-\mathrm{Na}_{4}$, and $15 \%$ acetonitrile $\mathrm{v} / \mathrm{v}$, pH 6.04). Amino acid separation was done using a Velosep RP-18 column $(3 \mu \mathrm{m} \times 100 \mathrm{~mm} \times 3.2 \mathrm{~mm}$; Perkin-Elmer $)$. A florescence spectrophotometer (RF-10A; Shimadzu) detected glutamate concentrations using an excitation wavelength of $340 \mathrm{~nm}$ and an emission wavelength of $450 \mathrm{~nm}$. A chart recorder recorded the peaks, and peak heights were measured manually and quantified using external glutamate standard curves as described previously (Melendez et al., 2005).

Histology. Rats were anesthetized and decapitated immediately after completion of the dialysis experiment, and the brains were cut and stained with thionine for histological verification of the cannulae and probe tracts.

Data analysis. Statistical analysis was determined by mixed factor ANOVAs that evaluated behavioral and microdialysis data followed by Tukey's honestly significant difference (HSD) tests when an interaction or main effect was found. Data from rats that failed microdialysis or HPLC analysis or had an inaccurate microdialysis probe placement were excluded in the final data set.

\section{Results}

\section{Histology}

In experiments 1 and 2, all rats had correct injector cannulae placements within the prelimbic or anterior cingulate regions of the dmPFC (Fig. 1); however, dialysis probes from two rats in experiment 1 and two rats in experiment 2 were outside the NAc, and the data from these subjects were excluded from analysis.

\section{Self-administration, extinction, and reinstatement}

All rats exhibited stable active (right) lever responding over the last $3 \mathrm{~d}$ of self-administration that did not differ between groups (Fig. 2). An ANOVA revealed no difference between groups (before intracranial infusions $)$ on active $\left(F_{(1,18)}=0.034 ; p>0.05\right)$ or inactive $\left(F_{(1,18)}=1.03 ; p>0.05\right.$; data not shown $)$ lever pressing during the last $3 \mathrm{~d}$ of self-administration. All rats underwent extinction training until criteria were met $(<25$ active lever presses per day). As reported previously (Berglind et al., 2007), there was a significant effect of intra-PFC BDNF treatment to reduce active $\left(F_{(1,18)}=3.434 ; p<0.05\right)$, but not inactive $\left(F_{(1,18)}=\right.$ $0.001 ; p>0.05$; data not shown), lever responding during the 


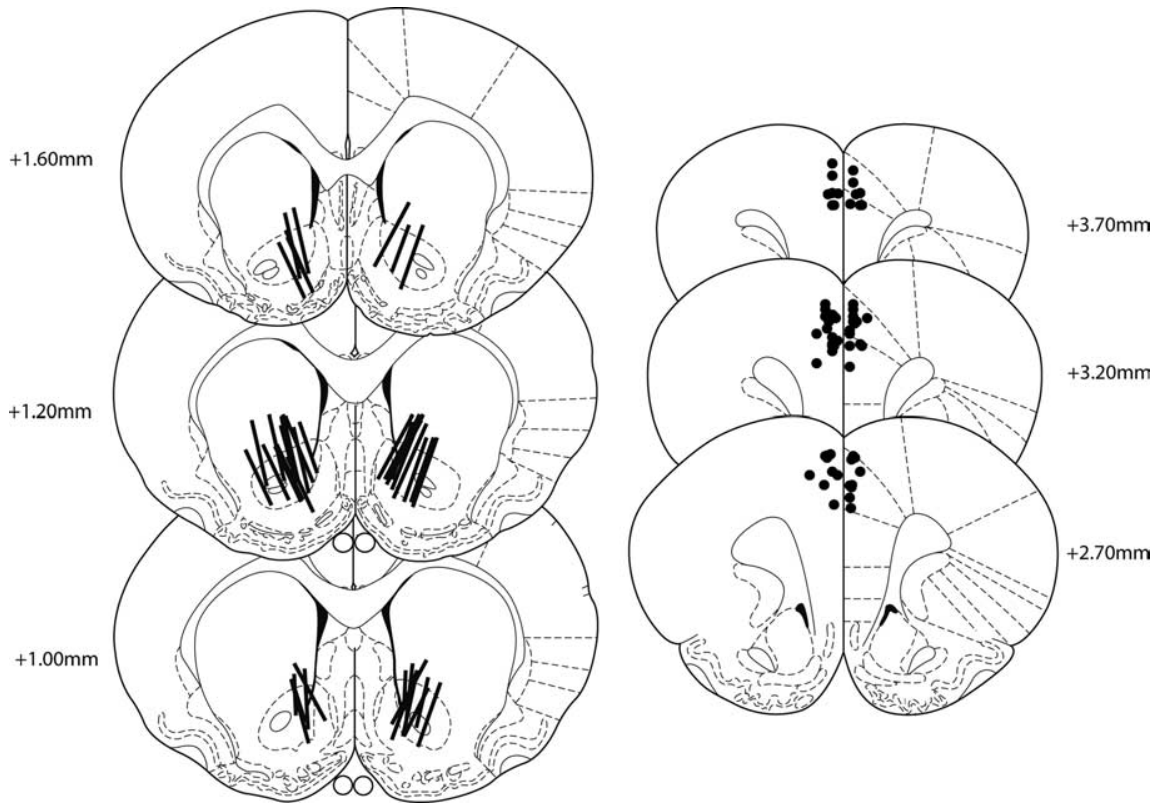

Figure 1. Cannula placements. A schematic representation of the active length of dialysis membranes within the NAc (left) and the tip of the infusion cannulae within the dmPFC (right).

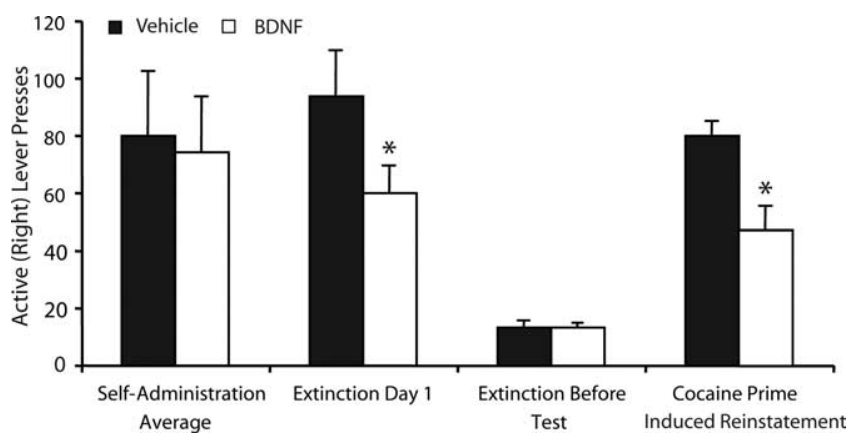

Figure 2. Intra-dmPFC BDNF suppressed cocaine-primed reinstatement. Left, The average number of lever presses during the last $3 \mathrm{~d}$ of self-administration (before BDNF infusion) was not different between groups ( $N=9-10$ per group). Intra-PFC BDNF infusion at the end of self-administration suppressed active lever presses on the first day of extinction (left, middle) but did not affect the remainder of extinction training (right, middle). Intra-dmPFC BDNF suppressed cocaine prime-induced reinstatement (right, $N=6-8$ rats per group; only rats with complete microdialysis data were included). ${ }^{*} p<0.05$.

first day of extinction. However, there was no effect of intra-PFC BDNF treatment on active lever-pressing $\left(F_{(1,18)}=0.009 ; p>\right.$ $0.05)$, or inactive lever-pressing $\left(F_{(1,18)}=0.056 ; p>0.05\right.$; data not shown) during the last $2 \mathrm{~d}$ of extinction training before reinstatement testing (Fig. 2). At the end of extinction training, the rats in experiment 1 underwent a $2 \mathrm{~h}$ cocaine prime-induced reinstatement session. A one-way ANOVA revealed a significant reduction of active lever-pressing during the $2 \mathrm{~h}$ reinstatement test after intra-PFC BDNF treatment $\left(F_{(1,12)}=10.66 ; p<0.05\right)$ (Fig. 2) without an effect on inactive lever-pressing $\left(F_{(1,12)}=\right.$ 2.995; $p>0.05$; data not shown).

Experiment 1: intra-PFC BNDF normalized basal and blocked a cocaine prime-induced increase in extracellular levels of glutamate in the NAc

After extinction training, rats in experiment 1 underwent microdialysis procedures to evaluate extracellular glutamate levels within the NAc before and after a single cocaine injection (10 $\mathrm{mg} / \mathrm{kg}$, i.p.). Basal and cocaine-induced extracellular glutamate levels were measured for two h at $10 \mathrm{~min}$ intervals. Statistical analysis was determined by evaluating the area under the curve (AUC) for samples from each group for $2 \mathrm{~h}$ before and for $2 \mathrm{~h}$ after the cocaine injection. A mixedfactor ANOVA revealed a significant interaction of group $\times$ time $\left(F_{(1,18)}=4.798\right.$; $p<0.01)$. A Tukey's HSD test revealed a significant effect of intra-PFC BDNF treatment on the AUC after the cocaine prime $(p=0.01)$ but not during baseline collection $(p=0.77)$ (Fig. 3). Furthermore, a Tukey's HSD test revealed a significant effect of cocaine on glutamate dialysate levels compared with baseline levels in the vehicle-treated group $(p=0.01)$ but not in the BDNF-treated group $(p=0.969)$.

Experiment 2: intra-PFC BNDF blocked cocaine self-administration-induced suppression of basal levels of glutamate within the NAc

To quantify the basal levels of extracellular glutamate in the NAc more precisely, a no-net flux experiment was performed that demonstrated the ability of intra-PFC BDNF to normalize basal levels of extracellular glutamate within the NAc after cocaine self-administration. Figure 4 illustrates the gain or loss of glutamate as a function of the concentration of glutamate added to the perfusate (Lönnroth et al., 1987). Using linear regression analysis, the slope was not significantly different among groups $\left(F_{(3,27)}=1.24 ; p>0.05\right)$. However, there was a significant interaction between drug treatment (COC vs SAL) and infusion condition (BDNF vs VEH) $\left(F_{(3,27)}=158.3 ; p<\right.$ $0.05)$ in the value of the $x$-intercept, a measure that indicates the level of extracellular glutamate within the NAc. Pairwise comparisons (Tukey HSD) revealed that the COC-VEH group had a significantly lower $x$-axis intercept $(1.523 \pm 0.079)$ than the COC-BDNF (3.676 $\pm 0.38 ; p<0.001)$, SAL-VEH (2.602 \pm 0.42 ; $p<0.05)$, and SAL-BDNF (3.146 $\pm 0.652 ; p<0.05)$ groups (Fig. $4 b$ ), indicating that cocaine-treated rats had significantly lower basal extracellular glutamate levels within the NAc compared with saline-treated rats and cocaine self-administering rats that received intra-PFC BDNF. There was no significant difference between the COC-BDNF and SAL-BDNF $x$-axis intercept, indicating that the ability of cocaine to significantly diminish levels of extracellular glutamate in the NAc was not present in the rats that received intra-PFC BDNF. Furthermore, there was no significant difference between SAL-VEH or SAL-BDNF-treated rats, indicating that BDNF alone had no effect on basal levels of extracellular glutamate.

\section{Discussion}

In the present study, an intra-dmPFC infusion of BDNF normalized the basal levels and prevented a cocaine prime-evoked increase in the levels of extracellular glutamate in the NAc of rats with a cocaine self-administration history. Specifically, basal levels of glutamate within the NAc after extinction training were greater in intra-dmPFC BDNF-infused rats than in rats that received intra-PFC vehicle infusions. Furthermore, a cocaine prime-induced increase in extracellular glutamate in the NAc of vehicle-infused rats was completely prevented by intra-PFC in- 


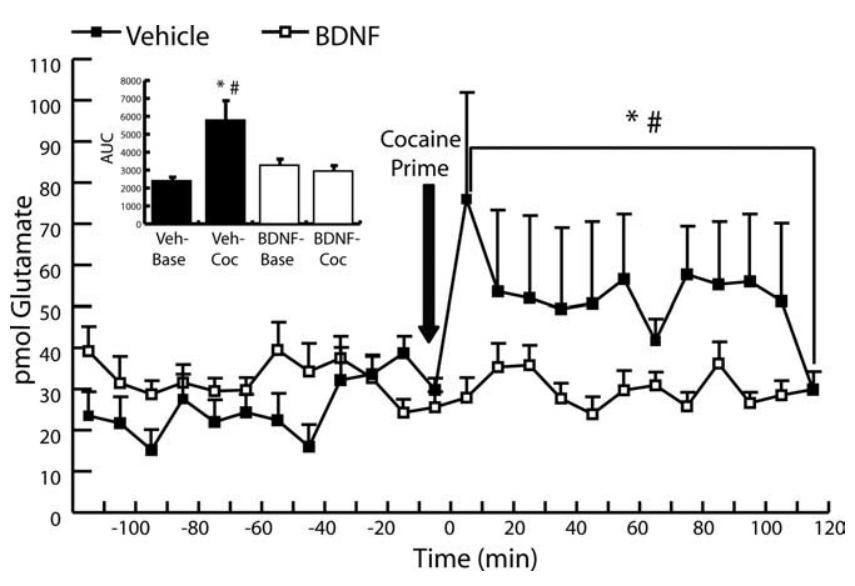

Figure 3. Effect of intra-dmPFC BDNF on extracellular glutamate levels in NAc before and after a single cocaine challenge $(10 \mathrm{mg} / \mathrm{kg}$, i.p.) in rats with a cocaine self-administration history. Microdialysis timeline at 10 min intervals before and after a cocaine challenge. Rats that received intra-PFC infusions of BDNF exhibited a suppression of the cocaine-induced increase in glutamate observed in rats that received intra-dmPFC vehicle infusions (VEH, $N=6 ; \mathrm{BDNF}, N=$ 8). ${ }^{*} p<0.05$, AUC of extracellular glutamate levels in BDNF-treated group after a cocaine prime versus AUC of extracellular glutamate levels in the vehicle-treated group after a cocaine prime; ${ }^{\#} p<0.05$, AUC of baseline extracellular glutamate levels in the vehicle-treated group versus extracellular glutamate levels in vehicle-treated group after a cocaine prime. Inset, Average AUC values before and after a cocaine challenge in rats with a cocaine history. VEHBase, Average baseline AUC value for rats that received an intra-dmPFC vehicle infusion; VEH$\triangle O C$, average AUC value after the cocaine challenge for rats that received a vehicle infusion; BDNF-Base, average baseline AUC value for rats that received an intra-dmPFC BDNF infusion; $B D N F-C O C$, average $A U C$ value after the cocaine challenge for rats that received an intra- $d m P F C$ BDNF infusion. ${ }^{*} p<0.05$ versus BDNF-COC; ${ }^{*} p<0.05$ versus VEH-Base.

fusion of BDNF at the end of self-administration training. Although these data are correlative, they strongly suggest that by normalizing prefrontal-accumbens glutamate transmission, BDNF suppresses cocaine seeking. Indeed, extensive evidence in rodent models of relapse to drug seeking indicate that the prefrontal-accumbens glutamatergic system is dysfunctional; glutamate levels in the NAc increase during reinstatement (Miguéns et al., 2008), and glutamate receptor activation is necessary for reinstatement to drug seeking (McFarland and Kalivas, 2001; Baker et al., 2003; McFarland et al., 2003; LaLumiere and Kalivas, 2008).

There are several possible explanations for the actions of intraPFC infused BDNF on NAc extracellular glutamate levels. First, it is likely that exogenous BDNF binds to TrkB receptors and is taken up by, and transported throughout, neurons in the PFC where it can affect several intracellular compartments in the dendrites, soma, and axons (Kohara et al., 2001). Moreover, exogenous BDNF evokes action potentials in cortical neurons by activating a TrkB-dependent sodium ion conductance (Kafitz et al., 1999). In this way, BDNF may increase glutamate exocytosis from $\mathrm{PFC}-\mathrm{NAc}$ neurons and restore extracellular glutamate tone by stimulating mGluR2/3 receptors, bypassing dysfunctional cystine/glutamate exchange (Moran et al., 2005). Mechanistically, exogenous BDNF activates presynaptic TrkB receptors and enhances depolarization-induced glutamate release from cortical synaptosomes via ERK-dependent phosphorylation of synapsin (Jovanovic et al., 2000) and enhances depolarization-evoked, glutamate exocytosis by activation of PLC, which induces the release of intracellular $\mathrm{Ca}^{2+}$ stores in cultured cortical neurons (Matsumoto et al., 2001). Alternatively, BDNF may interact with $\mathrm{xc}$ - to restore nonsynaptically derived extracellular glutamate levels in NAc. However, a direct interaction between antero-
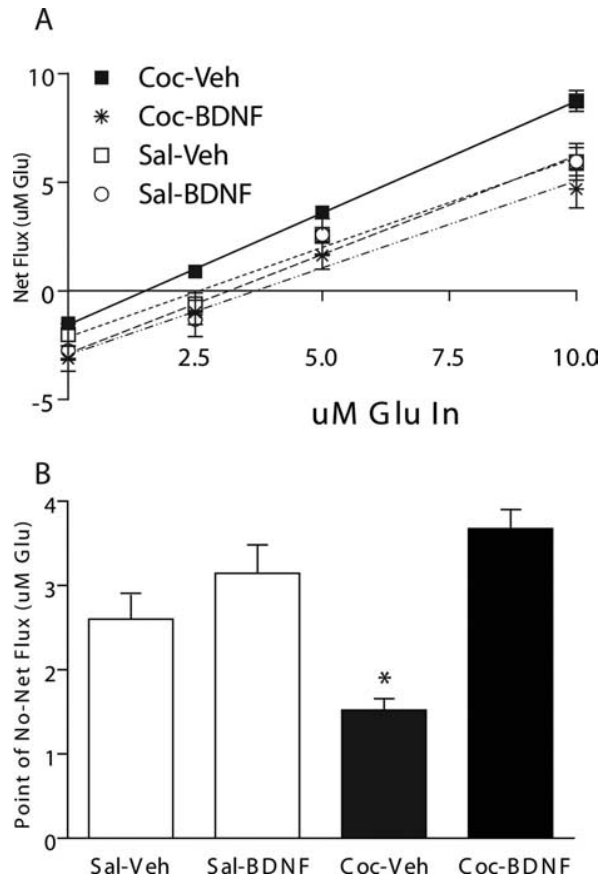

Figure 4. No-net flux procedure reveals that BDNF normalizes basal NAc glutamate levels in rats with a cocaine self-administration history. $A$, The mean $\pm($ SEM) gain or loss of dialysate glutamate concentrations to and from the brain as a function of the perfusate glutamate concentration. A positive number on the $y$-axis indicates a net diffusion of glutamate into the brain, and a negative number indicates a net diffusion from the brain into the perfusate. The zero point on the $y$-axis indicates the steady state at which there is no-net flux of glutamate across the dialysis membrane and provides a measure of the basal extracellular glutamate concentration in each group. $\boldsymbol{B}, \mathrm{BDNF}$ normalizes basal extracellular glutamate levels in the NAc of rats with a cocaine self-administration history. Intra-dmPFC BDNF treatment significantly increased the point of no-net flux, indicating an increase in the basal extracellular glutamate concentrations in the rats compared with those that received intra-dmPFC vehicle infusions. ${ }^{*} p<0.05(N=$ 4-7 per group).

gradely transported BDNF in PFC-NAc neurons and xc- expressed by local glia would require BDNF release and binding to TrkB receptors in the NAc. The unlikeliness of this explanation is provided by evidence that infusion of BDNF into the NAc augments cocaine seeking (Graham et al., 2007). Further studies will be needed to investigate the mechanism by which BDNF restores basal extracellular glutamate levels and prevents a cocaine primeinduced increase in glutamate in the NAc.

Intra-PFC BDNF appears to be able to stabilize the cocaineinduced depression in PFC activity and restore PFC-NAc glutamatergic tone. There is substantial evidence that repeated cocaine self-administration induces neuroadaptations within the PFC that lead to decreases in PFC-NAc neuronal activity. This hypoactivity has been demonstrated by decreases in glucose utilization proportional to the duration of self-administration (Macey et al., 2004) and a significant decrease in firing rates and membrane oscillations in PFC pyramidal neurons after repeated cocaine in rats (Trantham et al., 2002). Additionally, the hypoactive state during withdrawal from chronic cocaine stands in contrast to an exaggerated elevation in activity during re-exposure to cocainerelated stimuli or by cocaine itself, as recently demonstrated by Sun and Rebec (2006). They found that basal activity in the PFC was significantly decreased after repeated cocaine self-administration; however, there was a significant increase in burst firing rates in response to cocaine. These findings are consistent with the cocaine self-administration-induced decrease in basal NAc glutamate levels present immediately before daily self- 
administration sessions and after extinction training (Madayag et al., 2007; Miguéns et al., 2008). Also, the PFC burst firing response to a cocaine prime is consistent with increases in extracellular glutamate levels in the NAc of rats with a cocaine selfadministration history (Baker et al., 2003; McFarland et al., 2003; Madayag et al., 2007; Miguéns et al., 2008). Together, these studies substantiate that repeated cocaine suppresses basal activity and extracellular glutamate tone in the PFC-NAc pathway between drug exposures and causes hyper-responsive neuronal activity and glutamate neurotransmission in the PFC-NAc pathway when cocaine is administered. BDNF in the PFC appears to short-circuit this vicious cycle by normalizing PFC-NAc neurotransmission immediately after cocaine exposure.

In conclusion, elevating BDNF levels in the dmPFC immediately after the last exposure to cocaine restores the homeostatic balance of PFC-NAc neurotransmission that is disrupted by repeated cocaine and prevents relapse to cocaine seeking. Future studies using selective inhibitors of BDNF-activated signaling cascades in the prefrontal cortex and nucleus accumbens will determine which cascades mediate the ability of BDNF to normalize accumbens glutamate levels and suppress cocaine seeking.

\section{References}

Baker DA, Xi ZX, Shen H, Swanson CJ, Kalivas PW (2002) The origin and neuronal function of in vivo nonsynaptic glutamate. J Neurosci 22:9134-9141.

Baker DA, McFarland K, Lake RW, Shen H, Tang XC, Toda S, Kalivas PW (2003) Neuroadaptations in cystine-glutamate exchange underlie cocaine relapse. Nat Neurosci 6:743-749.

Bell K, Duffy P, Kalivas PW (2000) Context-specific enhancement of glutamate transmission by cocaine. Neuropsychopharmacology 23:335-344.

Berglind WJ, See RE, Fuchs RA, Ghee SM, Whitfield TW Jr, Miller SW, McGinty JF (2007) A BDNF infusion into the medial prefrontal cortex suppresses cocaine seeking in rats. Eur J Neurosci 26:757-766.

Capriles N, Rodaros D, Sorge RE, Stewart J (2003) A role for the prefrontal cortex in stress- and cocaine-induced reinstatement of cocaine seeking in rats. Psychopharmacology (Berl) 168:66-74.

Fuchs RA, Evans KA, Parker MC, See RE (2004) Differential involvement of the core and shell subregions of the nucleus accumbens in conditioned cue-induced reinstatement of cocaine seeking in rats. Psychopharmacology 176:459-465.

Fuchs RA, Evans KA, Ledford CC, Parker MP, Case JM, Mehta RH, See RE (2005) The role of the dorsomedial prefrontal cortex, basolateral amygdala, and dorsal hippocampus in contextual reinstatement of cocaine seeking in rats. Neuropsychopharmacology 30:296-309.

Graham DL, Edwards S, Bachtell RK, DiLeone RJ, Rios M, Self DW (2007) Dynamic BDNF activity in nucleus acccumbens with cocaine use increases self-administration. Nat Neurosci 10:1029-1037.

Jovanovic JN, Czernik AJ, Fienberg AA, Greengard P, Sihra TS (2000) Synapsins as mediators of BDNF-enhanced neurotransmitter release. Nat Neurosci 3:323-329.

Kafitz KW, Rose CR, Thoenen H, Konnerth A (1999) Neurotrophin-evoked rapid excitation through TrkB receptors. Nature 401:918-921.

Kalivas PW, Volkow ND (2005) The neural basis of addiction: a pathology of motivation and choice. Am J Psychiatry 162:1403-1413.

Kohara K, Kitamura A, Morishima M, Tsumoto T (2001) Activitydependent transfer of brain-derived neurotrophic factor to postsynaptic neurons. Science 291:2419-2423.

LaLumiere RT, Kalivas PW (2008) Glutamate release in the nucleus accumbens core is necessary for heroin seeking. J Neurosci 28:3170-3177.
Lessmann V (1998) Neurotrophin-dependent modulation of glutamatergic synaptic transmission in the mammalian CNS. Gen Pharmacol $31: 667-674$.

Lönnroth P, Jansson PA, Smith U (1987) A microdialysis method allowing characterization of intercellular water space in humans. Am J Physiol 253:E228-E231.

Losonczy A, Somogyi P, Nusser Z (2003) Reduction of excitatory postsynaptic responses by persistently active metabotropic glutamate receptors in the hippocampus. J Neurophsyiol 89:1910-1919.

Lu B (2003) BDNF and activity-dependent synaptic modulation. Learn Mem 10:86-98.

Lu L, Dempsey J, Liu SY, Bossert JM, Shaham Y (2004) A single infusion of brain-derived neurotrophic factor into the ventral tegmental area induces long-lasting potentiation of cocaine seeking after withdrawal. J Neurosci 24:1604-1611.

Macey DJ, Rice WN, Freedland CS, Whitlow CT, Porrino LJ (2004) Patterns of functional activity associated with cocaine self-administration in the rat change over time. Psychopharmacology (Berl) 172:384-392.

Madayag A, Lobner D, Kau KS, Mantsch JR, Abdulhameed O, Hearing M, Grier MD, Baker DA (2007) Repeated N-acetylcysteine administration alters plasticity-dependent effects of cocaine. J Neurosci 27:13968-13976.

Matsumoto T, Numakawa T, Adachi N, Yokomaku D, Yamagishi S, Takei N, Hatanaka H (2001) Brain-derived neurotrophic factor enhances depolarization-evoked glutamate release in cultured cortical neurons. J Neurochem 79:522-530.

McFarland K, Kalivas PW (2001) The circuitry mediating cocaine-induced reinstatement of drug-seeking behavior. J Neurosci 21:8655-8663.

McFarland K, Lapish CC, Kalivas PW (2003) Prefrontal glutamate release into the core of the nucleus accumbens mediates cocaine-induced reinstatement of drug-seeking behavior. J Neurosci 23:3531-3537.

McLaughlin J, See RE (2003) Selective inactivation of the dorsomedial prefrontal cortex and the basolateral amygdala attenuates conditioned-cued reinstatement of extinguished cocaine-seeking behavior in rats. Psychopharmacology (Berl) 168:57-65.

Melendez RI, Hicks MP, Cagle SS, Kalivas PW (2005) Ethanol exposure decreases glutamate uptake in the nucleus accumbens. Alcohol Clin Exp Res 29:326-333.

Miguéns M, Del Olmo N, Higuera-Matas A, Torres I, García-Lecumberri C, Ambrosio E (2008) Glutamate and aspartate levels in the nucleus accumbens during cocaine self-administration and extinction: a time course microdialysis study. Psychopharmacology (Berl) 196:303-313.

Moran MM, McFarland K, Melendez RI, Kalivas PW, Seamans JK (2005) Cystine/glutamate exchange regulates metabotropic glutamate receptor presynaptic inhibition of excitatory transmission and vulnerability to cocaine seeking. J Neurosci 25:6389-6393.

Paxinos G, Watson C (1998) The rat brain in stereotaxic coordinates. San Diego: Academic.

Pierce RC, Bell K, Duffy P, Kalivas PW (1996) Repeated cocaine augments excitatory amino acid transmission in the nucleus accumbens only in rats having developed behavioral sensitization. J Neurosci 16:1550-1560.

Schratt GM, Nigh EA, Chen WG, Hu L, Greenberg ME (2004) BDNF regulates the translation of a select group of mRNAs by a mammalian target of rapamycin-phosphatidylinositol 3-kinase-dependent pathway during neuronal development. J Neurosci 24:7366-7377.

Sun W, Rebec GV (2006) Repeated cocaine self-administration alters processing of cocaine-related information in rat prefrontal cortex. J Neurosci 26:8004-8008.

Trantham H, Szumlinski KK, McFarland K, Kalivas PW, Lavin A (2002) Repeated cocaine administration alters the electrophysiological properties of prefrontal cortical neurons. Neuroscience 113:749-753.

Xi ZX, Baker DA, Shen H, Carson DS, Kalivas PW (2002) Group II metabotropic glutamate receptors modulate extracellular glutamate in the nucleus accumbens. J Pharmacol Exp Ther 300:162-171. 